\title{
Sex differences in thoracic adaptation to pulmonary hyperinflation in cystic fibrosis
}

\author{
F. Bellemare* and A. Jeanneret ${ }^{\#}$
}

ABSTRACT: Sex differences in thoracic adaptation have been reported in patients with cystic fibrosis (CF). The interplay between the pattern of thoracic adaptation and the function of the respiratory muscles in male and female CF patients with pulmonary hyperinflation was investigated.

Thoracic dimensions and diaphragm length were measured at residual volume, functional residual capacity and total lung capacity using chest radiography in 23 CF (12 males) and 18 normal (11 males) subjects. Respiratory muscle recruitment during resting breathing was assessed by recording intrathoracic and intra-abdominal pressures.

In female CF patients, ribcage expansion was predominant, tending to preserve diaphragm length. In male CF patients, thoracic configuration was normal and diaphragm shortening consequently greater. Ribcage cross-sectional area for a given rib inclination was greater in CF patients, indicating a structural expansion of the ribcage $\mathbf{2 . 5}$ times greater in females than males. The contribution of inspiratory ribcage muscles to inspiratory pressure was also greater relative to the diaphragm in the CF group.

In conclusion, a structural expansion of the ribcage occurs in cystic fibrosis patients with lung hyperinflation that is greater in females than males. This is associated with an apparent greater contribution of inspiratory ribcage muscles to inspiratory pressure.

\section{KEYWORDS: Chest wall, chest radiography, diaphragm, sex, respiratory muscles}

$\mathbf{P}$ ulmonary hyperinflation, as occurs in cystic fibrosis (CF) patients, may impair thoraco-abdominal mechanics and the function of the inspiratory muscles [1, 2], factors that may contribute to dyspnoea and poor exercise tolerance [3]. A shortening of the inspiratory muscles, particularly the diaphragm [4-7], and a change in its geometry with pulmonary hyperinflation [6-8], are factors known to reduce the pressure-generating capacity of the inspiratory muscles. However, the effect of pulmonary hyperinflation on maximum inspiratory pressure in CF patients is controversial, some studies reporting a decrease $[1,2,9$, 10], others no change [11], and yet others an improvement in maximum inspiratory pressure in spite of pulmonary hyperinflation [12]. It is very difficult to reconcile these different results. In studies so far, the effect of pulmonary hyperinflation on diaphragm length (Ldi) and geometry has not been investigated in CF patients. Furthermore, whether the impact of pulmonary hyperinflation on Ldi and diaphragm function is the same in male and in female CF patients is also unknown.

In a study by LANDS et al. [11], maximum inspiratory pressure was reported to be better preserved in female than in male CF patients. Furthermore, in a selected group of CF patients with greater than normal lung volume at total lung capacity (TLC), this extra volume was shown to be distributed preferentially via the rib cage pathway in females but via the diaphragm-abdomen pathway in males [13]. These findings suggest that the effect of pulmonary hyperinflation on Ldi and diaphragm function may be different in males and females. Previous conclusions regarding thoraco-abdominal adaptation in CF patients are restricted to measurements made at TLC, and thus may not be extended to other lung volumes, particularly functional residual capacity (FRC), where this issue is most important. The primary objectives of the present study, therefore, were to compare the pattern of thoraco-abdominal adaptation at different lung volumes in a representative group of male and female CF patients and to investigate the effect this may have on Ldi and diaphragm function.

\section{METHODS}

\section{Subjects}

After approval by the human ethics committee of the Centre hospitalier de l'Université de Montréal-Hôtel Dieu, Montréal, QC, Canada, 23
AFFILIATIONS

*Laboratoire du sommeil, and

\#Département de pneumologie, Centre hospitalier de l'Université de Montréal (CHUM)-Hôtel-Dieu,

Montréal, QC, Canada.

CORRESPONDENCE

F. Bellemare

Laboratoire du sommeil

CHUM-Hôtel-Dieu

3840 rue St-Urbain

Montréal

QC

H2W 1 T8

Canada

Fax: 15144127178

E-mail: bellemare@videotron.qc.ca

Received:

April 012006

Accepted after revision:

August 042006

SUPPORT STATEMENT

The contribution of Medigas,

St-Laurent, OSR Medical, Montreal, and of the Laboratoires Biron Brassard, all Canada to the Foundation supporting F. Bellemare is gratefully acknowledged. 
stable CF patients and 18 age- and sex-matched normal subjects were studied (table 1).

\section{Chest radiography}

Chest radiographs served to measure thoracic dimensions and Ldi. Details of the techniques and measurements can be found in recent publications $[7,14,15]$. Briefly, subjects stood erect with their heel, calf, buttock, back and head against a vertical backboard and with the head position fixed with a strap. Lateral and anterior-posterior chest radiographs were taken in this position at the end of a normal expiration (i.e. FRC), at the end of a full inspiration to TLC and at the end of a full expiration to residual volume (RV).

\section{Chest wall configuration}

The mean internal anterior-posterior (APrc) and lateral (LArc) ribcage diameters at the level of the third, fifth, seventh and ninth thoracic vertebrae and ribs, respectively, were measured and their product used to estimate ribcage cross-sectional area (Arc), assuming a cross-sectional shape one-third of the way between an ellipse and a rectangle [16, 17]. The rib cage was also divided into an upper (U) and a lower (L) segment defined by the mean diameters and cross-sectional areas at the levels of the 3rd and 5th thoracic vertebrae and ribs (APrc_U, LArc_U, Arc_U) and at the level of the 7th and 9th thoracic vertebrae and ribs, respectively (APrc_L, LArc_L, Arc_L). The acute angle formed by the lower border of the sixth rib and the vertical on lateral films and the average height of the two hemidiaphragm domes (Hdi) below the first thoracic vertebra on anterior-posterior films were also measured [15].

\section{Diaphragm length}

Ldi was measured from diaphragm contours on the right-hand side using anatomic landmarks to identify diaphragmatic insertions on the chest wall and divided into the lengths of the visible contours and the length of that part of the diaphragm directly apposed to the ribcage [4, 14]. A dome shape factor was calculated as the ratio between the length of the visible contours and the length of chords intersecting contours' endpoints [18]. All linear dimensions were normalised to standing height. Furthermore, a correction factor of 0.9 was applied to all linear dimensions for the magnification of chest wall structure seen in chest radiography [4, 14].

\section{Respiratory muscle recruitment}

In all normal subjects and in $16 \mathrm{CF}$ patients who tolerated this procedure, oesophageal $(P$ oes $)$, gastric $(P$ ga $)$ and transdiaphragmatic $(P \mathrm{di})$ pressures were recorded with balloon-tipped catheters (all normal subjects and eight CF patients) or with smaller liquid-filled catheters (eight CF patients) [19] during 12 min of quiet breathing. Lung volume changes were recorded by integrating the mouth flow signal, measured with a pneumotachograph connected to a flanged mouthpiece and with a nose-clip in place. Tidal volume and tidal inspiratory $P$ oes, $P$ ga and $P$ di swings were measured between points of zero flow taken at beginning and end of inspiration. Pressure-time integrals above baseline were measured from the beginning of inspiration to the end of expiration and multiplied by breathing frequency.

\section{Statistical analysis}

Variables that satisfied Levene's test were compared using ANOVA or ANCOVA. Otherwise, the Mann-Whitney U-test was employed. A p-value $<0.05$ was considered statistically significant.

\section{RESULTS \\ Subject characteristics}

The two groups did not differ significantly with respect to age, height or sex distribution (table 1). CF patients had

TABLE 1 Physical and pulmonary function characteristics of subjects

\begin{tabular}{|c|c|c|c|c|}
\hline & \multicolumn{2}{|c|}{ Normal subjects } & \multicolumn{2}{|c|}{ Cystic fibrosis patients } \\
\hline & Males & Females & Males & Females \\
\hline Patients n & 11 & 7 & 12 & 11 \\
\hline Age yrs & $28 \pm 7.4(21-43)$ & $25.3 \pm 6.9(20-38)$ & $26.2 \pm 7.2(20-40)$ & $21.8 \pm 2.2(18-25)$ \\
\hline Height $\mathrm{cm}$ & $169.2 \pm 3.8$ & $166.1 \pm 5.2$ & $171 \pm 6.7$ & $160.6 \pm 7.1$ \\
\hline FVC \% pred & $110.6 \pm 12.5$ & $107.4 \pm 10.2$ & $72.6 \pm 17^{*}$ & $76.2 \pm 18^{*}$ \\
\hline FEV $1 \%$ pred & $108.9 \pm 15.5$ & $105.7 \pm 9.8$ & $47.3 \pm 16^{*}$ & $55.6 \pm 17.6^{*}$ \\
\hline TLC \% pred & $109.3 \pm 9.9$ & $103.1 \pm 11.4$ & $110.8 \pm 7.8$ & $111.7 \pm 11.8$ \\
\hline FRC \% pred & $108.9 \pm 23.2$ & $103.4 \pm 15$ & $146.8 \pm 19.7^{*}$ & $133.1 \pm 19.3^{*}$ \\
\hline RV \% pred & $106.4 \pm 33.4$ & $95.1 \pm 34.3$ & $232.3 \pm 56.7^{*}$ & $226.6 \pm 64.4^{*}$ \\
\hline Raw \% pred & $118.1 \pm 23.2$ & $105.1 \pm 35.9$ & $224.6 \pm 71.1^{*}$ & $181.9 \pm 74.6^{*}$ \\
\hline
\end{tabular}

Data are presented as group mean \pm SD (range) or group mean \pm SD, unless otherwise stated. All measures were obtained with the MedGraphic Elite Series Plethysmograph (Medgraphic Inc, St Paul, MN, USA). Lung volumes and airway resistance (Raw) were determined plethysmographically with a panting frequency of $\sim 120$ breaths $\cdot \mathrm{min}^{-1}$ (range: $90-120 \mathrm{~min}^{-1}$ for functional residual capacity (FRC) determination and $100-160$ breaths $\cdot \mathrm{min}^{-1}$ for Raw determination). IBW: ideal body weight; FVC: forced vital capacity; \% pred: \% predicted; FEV1: forced expiratory volume in one second; TLC: total lung capacity; RV: residual volume; DL,CO: single-breath diffusing capacity of the lung for carbon monoxide; VA: alveolar volume. *: $p<0.05$ versus normal subjects (Mann-Whitney U-test). 
significantly lower body weights and severe signs of airway obstruction and hyperinflation, as exemplified by marked increases in airway resistance and RV, respectively. FRC was also significantly greater in CF patients, but TLC and diffusing capacity of the lung for carbon monoxide were not significantly different from control values.

\section{Thoracic dimensions and configuration}

Thoracic dimensions are listed in table 2 and thoracic configuration illustrated in figure 1. The relationship between Arc and $\mathrm{Hdi}$ in male $\mathrm{CF}$ patients closely followed that in the controls (fig. 1a), indicating an essentially normal distribution of pulmonary hyperinflation between the ribcage and the diaphragm-abdomen pathways. By contrast, in female CF patients, this relationship was shifted upward, indicating a change in thoracic configuration and a preferential expansion of the rib cage at all lung volumes investigated (fig. 1b).

The relationship between APrc and LArc in female CF patients mirrored that in normal females, indicating a nondistorted ribcage (fig. 1d; ANCOVA; $p>0.5$ ). By contrast, this relationship was shifted upward and to the left in male CF patients compared with controls (ANCOVA; $\mathrm{p}<0.001$ ), indicating a distorted, more round ribcage (fig. 1c).
As shown in table 2, rib angles were not significantly different between the two groups. However, as seen in figure 1e and $f$, the relationship between Arc and rib angle was shifted upward and to the left in CF patients compared with controls. This shift for the group was highly significant, suggesting a structural enlargement of the bony ribcage in CF patients (ANCOVA; $\mathrm{p}<0.001$ ). In female CF patients, this shift corresponds to a $13 \%$ increase in Arc at a given rib angle (fig. 1f; ANCOVA; $p<0.001$ ), whereas in males this increase was only $5.3 \%$ (fig. $1 \mathrm{e}$; ANCOVA; $\mathrm{p}=0.1$ ). When indexes of pulmonary hyperinflation (\% predicted RV, FRC or TLC) and airway obstruction (forced expiratory volume in one second (FEV1)/forced vital capacity (FVC)), were entered as covariates in the ANOVA model, all were significant (all $\mathrm{p}<0.0007$ ). Furthermore, in the case of \% pred RV and FRC and FEV1/FVC, the betweengroup difference in Arc at a given rib angle was no longer significant, indicating that the observed difference between normal subjects and CF patients was dependent on the severity of lung disease.

The relationships between upper and lower ribcage dimensions are illustrated in figure 2. In both male and female CF patients, the relationship between $A P_{r c} \_L$ and $A P r c \_U$ was shifted to the left of that seen in normal subjects (ANCOVA;

\section{TABLE 2 Thoracic configuration and diaphragm length}

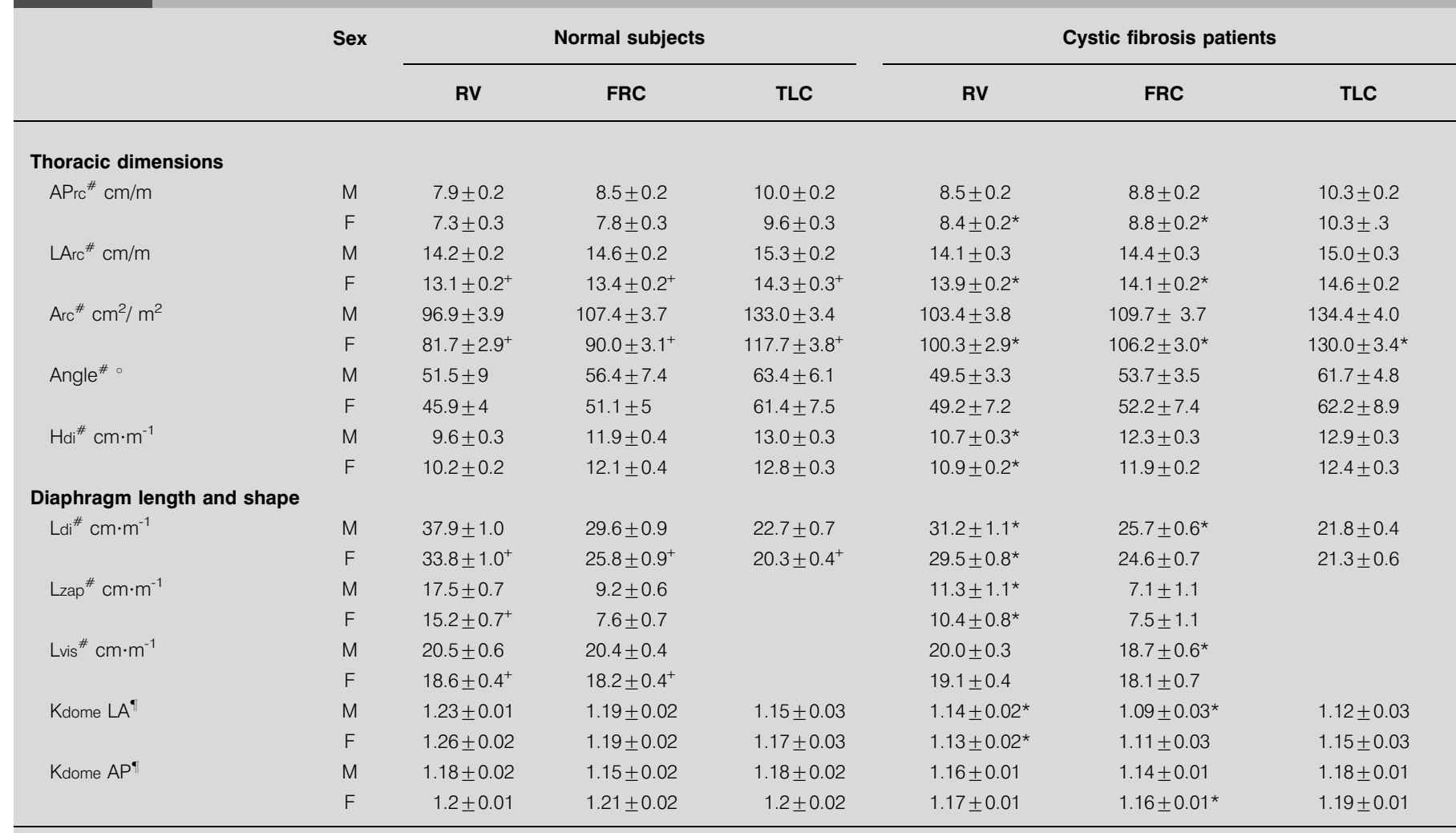

Data are presented as mean $\pm \mathrm{SEM} . \mathrm{cm} \cdot \mathrm{m}^{-1}$ and $\mathrm{cm}^{2} \cdot \mathrm{m}^{-2}$ denote normalisation for standing height. RV: residual volume; FRC: functional residual capacity; TLC: total lung capacity; APrc: anterior-posterior ribcage diameter; LArc: lateral ribcage diameter; Arc: ribcage cross-sectional area; Angle: angle between the lower border of the sixth rib and the vertical; Hdi: diaphragm dome height; Ldi: diaphragm length on the right-hand side; Lzap: length of the diaphragm apposed zone on the right-hand side; Lvis: length of the visible contour of the diaphragm on the right-hand side; Kdome LA: diaphragm dome shape factor on lateral projections; Kdome AP: diaphragm dome shape

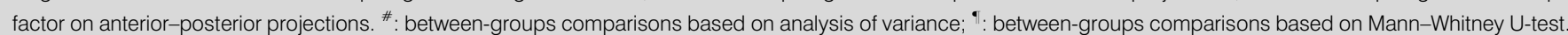
*: $p<0.05$ versus normal values $(p<0.05) ;{ }^{+}: p<0.05$, males versus females 

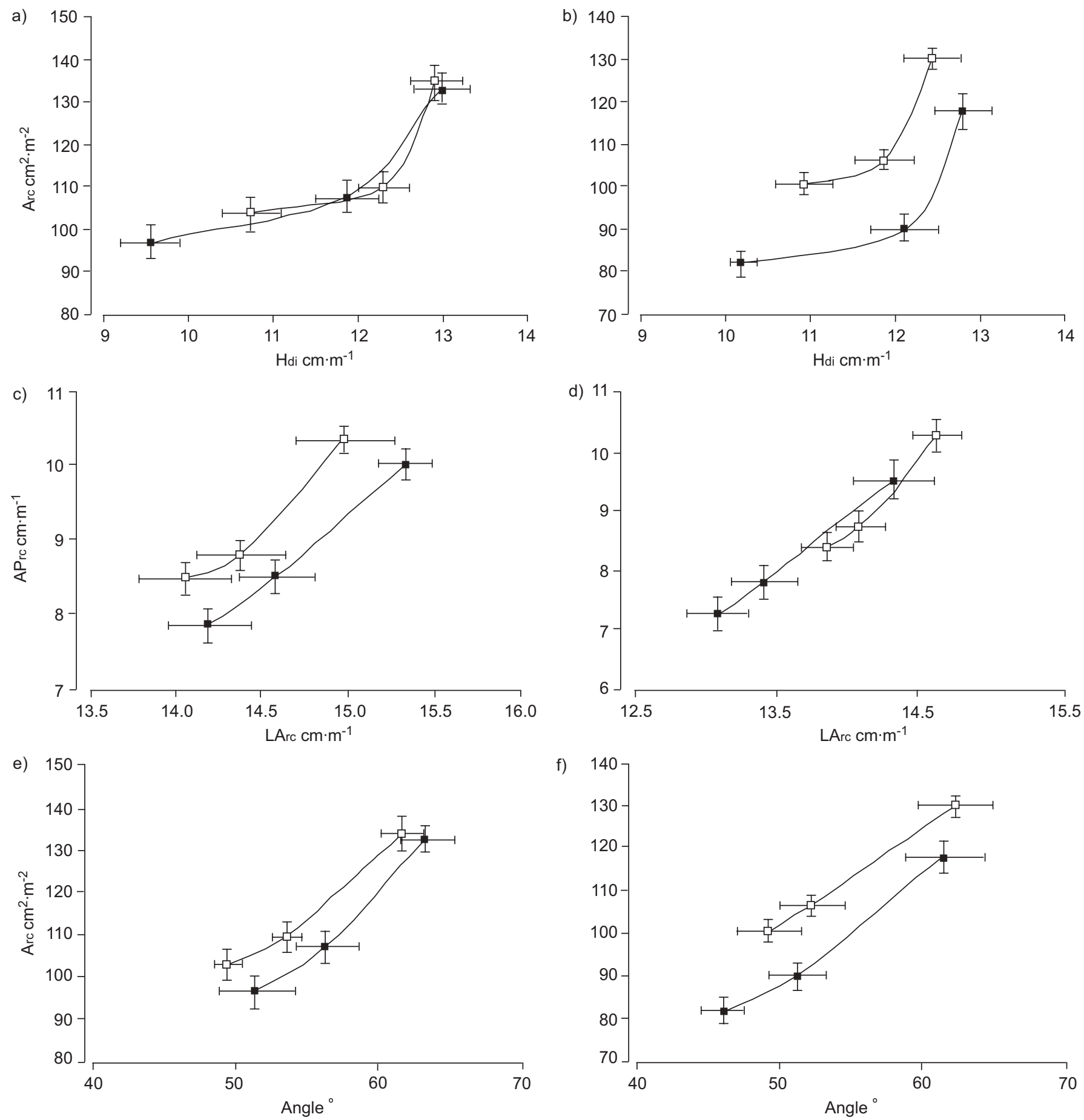

FIGURE 1. Thoracic configuration in male (a, c, e) and female (b, d, f) cystic fibrosis patients ( $\square$ ) and normal subjects ( $\mathbf{\square})$. Data are presented as group mean \pm SEM. Arc: ribcage cross-sectional area; Hdi: diaphragm dome height; APrc: ribcage anterior-posterior diameter; LArc: ribcage lateral diameter; Angle: angle between the lower border of the sixth rib and the vertical.

$\mathrm{p}<0.003)$, whereas that between LArc_L and LArc_U was shifted to the right (ANCOVA; $\mathrm{p}=0.02$ ), indicating a cranio-caudal deformation of the rib cage. In spite of this deformation, the relationship between Arc_L and Arc_U in male CF patients was not significantly different from that seen in normal subjects (ANCOVA; $p>0.1$ ). In female CF patients, however, the relationship between Arc_L and Arc_U was shifted upward and to the left of that seen in normal females (ANCOVA; $p=0.005)$, indicating a relatively greater expansion of the lower ribcage.

\section{Diaphragm length}

Diaphragm shortening with lung hyperinflation in the CF group can be estimated as the between-group difference in Ldi 
at a given lung volume using the values listed in table 2 . Estimated this way, mean diaphragm shortening at RV and FRC was 1.4 and 2.5 times greater, respectively, in male than in female CF patients. Of note, Ldi in female CF patients was not significantly shorter at FRC than in controls and tended to be, if anything, slightly longer at TLC. Diaphragm shortening in CF patients was largely explained by a shortening of the length of the rib cage-apposed zone, the changes in the length of the visible contours being less consistent. Several dome shape factors at RV and FRC were significantly smaller in the CF patients than in the controls, indicating a flatter diaphragm.

\section{Respiratory muscle recruitment}

End-inspiratory pressure swings and inspiratory pressuretime integrals during resting breathing in the two groups are listed in table 3 . In figure 3 , end-inspiratory $P$ oes and $P$ ga swings during resting breathing are shown in relation to tidal volume, expressed as \% pred vital capacity. End-inspiratory Poes swings were more negative in relation to tidal volume in CF patients than in control subjects, indicating a smaller dynamic lung compliance. End-inspiratory Pga swings also tended to be less positive in CF patients than in normal subjects, though the difference was not statistically significant $(p=0.08)$. However, the ratios of end-inspiratory $P_{\text {oes }}$ to $P$ di swings and the ratio of inspiratory $P_{\text {oes }}-$ to $P$ di-time integrals were significantly greater (i.e. more negative) in CF patients than in normal subjects (table 3), suggesting a greater contribution of inspiratory ribcage muscles relative to the diaphragm in the CF group (ANOVA; $p<0.001$ ) [20]. For both groups, end-inspiratory Pga swings were significantly smaller and the ratio of end-inspiratory $P$ oes to $P$ di swings significantly greater (i.e. more negative) in females than in males, indicating a stronger recruitment of inspiratory ribcage muscles relative to the diaphragm in females (ANOVA; $\mathrm{p}<0.002$ ). A similar trend was found for the ratio of inspiratory Poes- to Pdi-time integrals, but this difference was not statistically significant $(p=0.09)$.

Expiratory recruitment of abdominal muscles during resting breathing, as reflected by an increase in gastric pressure at the beginning of expiration and a decrease just before or at the beginning of the next inspiration, was found in one normal male subject and in six CF patients (three males).

\section{DISCUSSION}

The present study shows systematic differences in the pattern of thoracic adaptation to pulmonary hyperinflation in male and in female CF patients at all lung volumes investigated. In female CF patients, increases in ribcage dimensions were predominant and were associated with a relative preservation of Ldi. In males, the changes in thoracic dimensions were more uniform along the ribcage and the diaphragm-abdomen pathways and were associated with a greater shortening of the diaphragm. Surprisingly, the preferential expansion of the ribcage in female CF patients was not associated with a greater elevation of the ribs. At any given rib angle, ribcage dimensions were greater in the CF group, suggesting an enlargement of the bony ribcage. This difference was about 2.5 times greater in females, thus explaining the sex difference in thoracic adaptation in CF patients. The pattern of respiratory pressure changes during resting breathing was indicative of a stronger contribution of inspiratory ribcage muscles relative to the diaphragm in CF patients and, for both groups, also suggestive of a relatively greater contribution of these muscles relative to the diaphragm in females than in males.

\section{Thoraco-abdominal configuration}

It has been shown previously that, after adjustment for height, the volume of the rib cage at any given lung volume is smaller in females than in males and that this is explained by a greater inclination of the ribs in females [15]. As a result, the relationship between Arc and Hdi in females was shifted to the right of that in males, causing the diaphragm to be shorter in relation to height in females. As the present study shows, these sex differences in thoraco-abdominal configuration and Ldi essentially disappeared in the CF group, owing to a greater expansion of the ribcage in female than in male CF patients (table 2). A greater ribcage expansion in females than in males was anticipated based on previous findings in a selected group of $\mathrm{CF}$ patients having greater than normal lung volumes at TLC [13]. Contrary to expectation, however, this preferential expansion of the ribcage in female CF patients was not associated with a greater elevation of ribs. Rather, as the results in figure $1 \mathrm{e}$ and $f$ suggest, the volume of the ribcage appeared to be greater in CF patients, presumably owing to a structural enlargement of the ribcage. This enlargement of the ribcage was 2.5 times greater in females than in males, thereby explaining the sex difference in thoracic adaptation in the CF group.

Two different mechanisms may be considered to explain this apparent increase in the volume of the rib cage in CF patients. Everything else being kept constant, an increase in Arc for a given inclination of ribs should reflect an elongation of ribs and/or costal cartilages. However, because the ribs lie in a three-dimensional space, rib angles measured on two-dimensional images must be interpreted with reservation. Indeed, the ribs rotate about a posterior axis forming an acute angle of about $35^{\circ}$ with the mid-sagittal plane [21]. This axis of rotation has two principal components, one perpendicular to the midplane causing APrc changes, and one located in the mid-plane causing LArc changes. These two components of rib rotation are usually described as the "pump handle" and "bucket handle" motions of ribs, respectively. The rib angle measured on lateral chest radiographs is a measure of the pump handle angle. In fact, the angles of the sixth rib in normal male subjects in the present study at FRC $\left(56.4^{\circ}\right)$ and TLC $\left(63.4^{\circ}\right)$ are very close to the pump handle angle calculated for rib planes by WILSON et al. [21] in two normal male subjects studied at comparable lung volumes (55.4 and $62^{\circ}$, respectively). The bucket handle angle of ribs cannot be estimated from standard chest radiographs. It is conceivable that a dissociation between the pump and bucket handle motions of ribs could occur in CF patients, causing a relatively greater bucket handle motion of ribs and, hence, a greater Arc for a given pump handle angle, as in figure $1 \mathrm{e}$ and $\mathrm{f}$. However, the present authors think this is unlikely for the following reasons. First, such a dissociation would entail a change in the axis of rotation of the ribs and a substantial deformation at the costovertebral joints. Secondly, everything else being constant, a dissociation between the pump and bucket handle motions of ribs would change the shape of the ribcage. Specifically, this would shift the relationship between APrc and LArc to the right, which is not what was found. In females, this relationship was essentially 

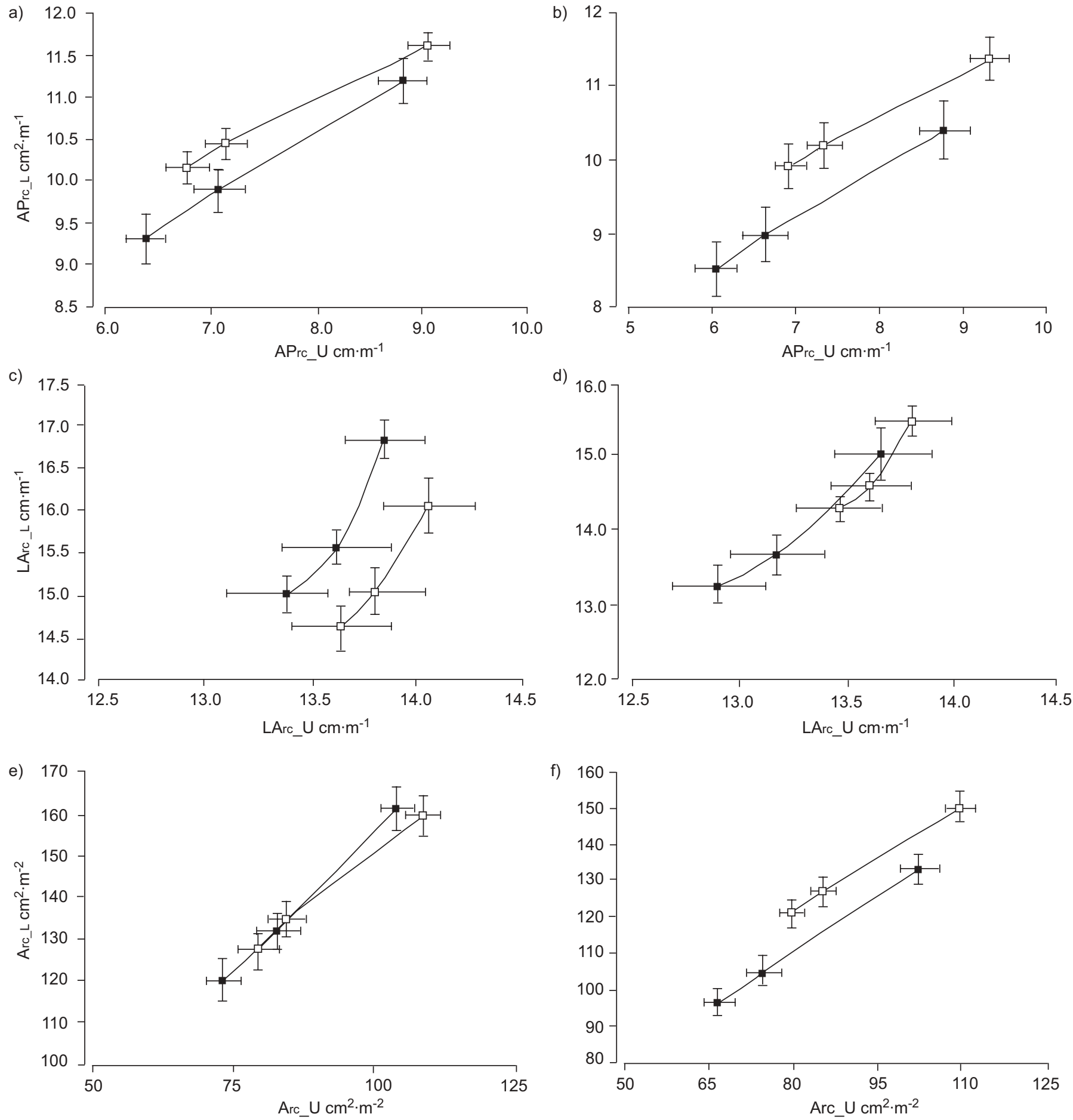

FIGURE 2. Relationships between upper and lower ribcage dimensions in male (a, c, e) and female (b, d, f) cystic fibrosis patients ( $\square$ ) and normal subjects ( $\mathbf{\square})$. Data are presented as group mean \pm SEM. APrc_L: lower ribcage anterior-posterior diameter; APrc_U: upper ribcage anterior-posterior diameter; LArc_L: lower ribcage lateral diameter: LArc_U: upper ribcage lateral diameter; Arc_L: lower ribcage cross-sectional area; Arc_U: upper ribcage cross-sectional area.

unchanged (fig. 1d) and in male CF patients, it was shifted to the left, i.e. in a direction opposite to that which would be anticipated for a relatively greater bucket handle motion of ribs (fig. 1c). Hence, a dissociation between the pump and bucket handle motions of ribs cannot explain the kind of shift seen. Only an elongation of the ribs can reasonably explain this finding.
THOMAs et al. [22] have shown, using the hamster model of elastase-induced emphysema, that for a degree of pulmonary hyperinflation comparable to that in $\mathrm{CF}$ patients in the current study, the lengths of the sternum and ribs are $\sim 10 \%$ greater in emphysematous animals than in controls. Since significant pulmonary hyperinflation can occur during the period of 
TABLE 3 Respiratory muscle recruitment

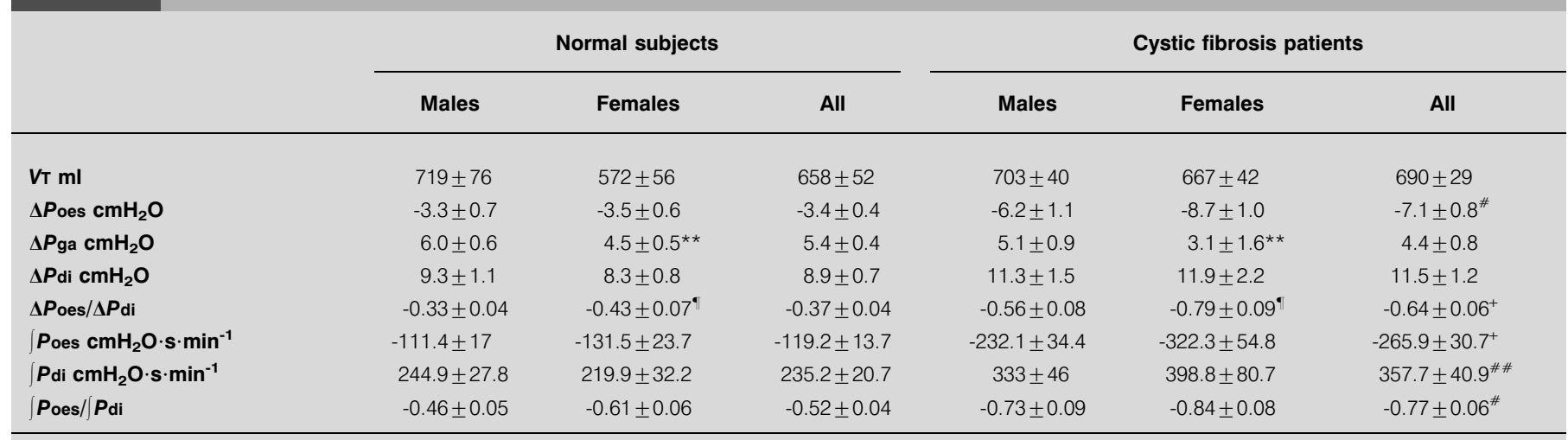

Data are presented as group mean \pm SEM. $V$ T: tidal volume; $\Delta P$ oes: change in tidal oesophageal pressure; $\Delta P$ ga: change in tidal gastric pressure; $\Delta P$ di: change in tidal transdiaphragmatic pressure; $(P$ es: oesophageal pressure-time integral multiplied by breathing frequency; $[P$ di: transdiaphragmatic pressure-time integral multiplied by breathing frequency. ${ }^{*}: p<0.005$ versus normal group (ANOVA); ${ }^{* *}: p<0.01$ versus males (ANOVA); ${ }^{\uparrow}: p<0.005$ versus males (ANOVA); ${ }^{+}: p<0.0005$ versus normal group (ANOVA); ${ }^{\# \#: ~} \mathrm{p}<0.01$ versus normal group (ANOVA)

growth in CF patients, the ribs and sternum may have grown longer in response to lung hyperinflation, as in the hamster model of emphysema. In support of this hypothesis is the finding of significantly longer sternum lengths in children with CF than in normal children having the same height [23]. In a more recent study, however, no significant difference was found in the length of the sixth rib and sternum between a group of adult CF patients and a group of normal subjects [24]. It should be taken into account that in that study, all subjects were males, which, as the results shown in figure 1e and $\mathrm{f}$ suggest, could explain the different results. In the earlier study in children, no distinction was made between male and female CF patients. However, the fact that both males and females were included could explain their different results. Further

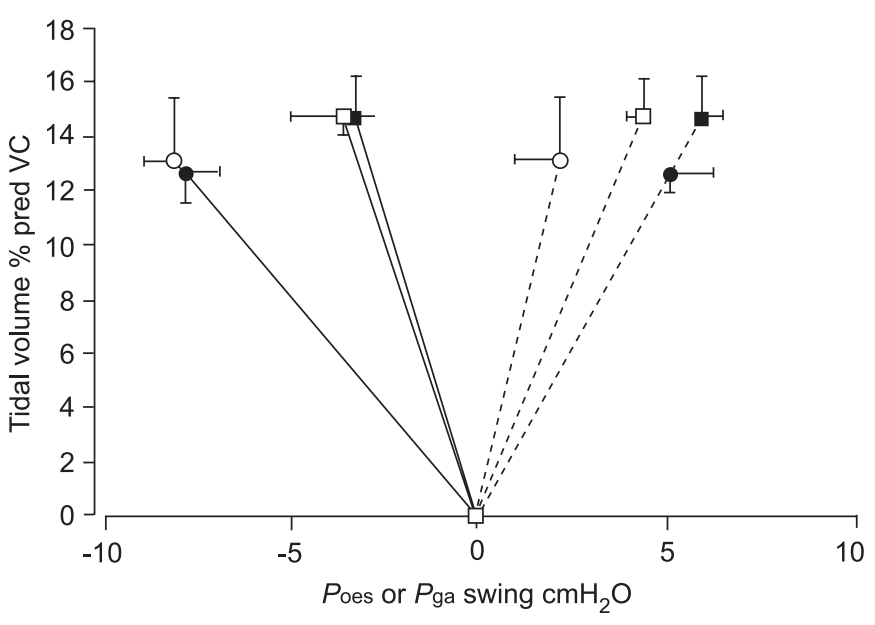

FIGURE 3. Relationship between tidal volume, expressed as \% predicted (\% pred) vital capacity (VC) and oesophageal $\left(P_{\text {oes; }}-\right.$ - ) or gastric pressure (Pga; .......) swings measured at points of zero flow in male $(\bullet)$ and female $(\bigcirc)$ patients with cystic fibrosis and in male ( $\mathbf{\square})$ and female $(\square)$ normal subjects. Data are presented as group mean \pm SEM. studies comparing male and female CF patients are, therefore, warranted. Such studies are planned.

The reason for the sex difference in ribcage adaptation to pulmonary hyperinflation in CF patients is unclear. A sexual dimorphism of human ribs was recently demonstrated, in that the ribs were shown to grow longer in relation to height in females compared to males [25]. Furthermore, in contrast to males, the lengths of ribs in females were not correlated with the length of the axial skeleton, suggesting that different mechanisms govern rib growth in males and in females. It is conceivable that in the presence of pulmonary hyperinflation, the growth factors responsible for this sexual dimorphism of human ribs could favour a greater elongation of ribs in female than in male CF patients. In male CF patients, the ribcage was deformed and rounder than in normal controls, whereas no such deformation was found in female CF patients (fig. 1c and d). It is also conceivable, therefore, that ribcage growth could somehow be impaired in male CF patients, which might explain this deformation as well as the smaller increase in Arc for a given inclination of ribs than in female CF patients. Interestingly, rib-cage expansion in female $\mathrm{CF}$ patients and rib-cage deformation in male CF patients appeared to be relatively greater at the level of the lower rib cage, suggesting that the upper and lower parts of the rib cage may be affected differentially by hyperinflation. Whether a chronic state of hyperinflation is required or whether these adaptations can also manifest themselves with periodic episodes of acute lung hyperinflation, as in asthma, is presently unknown.

\section{Implications for respiratory muscle function}

Pulmonary hyperinflation leads to the prediction of shorter diaphragm length $[4,6,7]$. In line with this prediction, diaphragm length at RV was significantly smaller in CF patients than in normal subjects. Contrary to this prediction, however, diaphragm length at FRC was not significantly different between the two groups, in spite of significant differences in FRC volume. This was particularly striking in female CF patients, in whom diaphragm length at FRC was 
essentially the same as in normal females and tended if anything to be even slightly longer at TLC (table 2). An elongation of ribcage bones substantially greater in females than in males may explain these findings. Indeed, because the diaphragm has its insertions on the sternum anteriorly and on the lower six ribs and costal cartilages laterally, an elongation of these structures would tend to increase Ldi and in this way protect the diaphragm against excessive shortening. Although the underlying mechanism is presently unknown, the relatively greater expansion of the lower ribcage in female $\mathrm{CF}$ patients should contribute to the explanation of this finding.

Ldi is an important determinant of the pressure-generating capacity of the diaphragm [4,5]. An elongation of the bony ribcage, by protecting the diaphragm from excessive shortening, may therefore be expected to also preserve $P$ di generation, particularly in female CF patients. Contrary to this prediction, however, the contribution of the diaphragm to inspiratory pressure was found to be reduced in CF patients, a reduction which, if anything, appeared to be accentuated in females (fig. 3). Several neural and mechanical factors may contribute to the explanation for this finding. The distribution of inspiratory neural drive between the inspiratory ribcage muscles and the diaphragm could not be measured, but could have been different in CF patients than in normal subjects. Indeed in contrast to the diaphragm, the ribcage muscles are richly supplied with muscle spindles. In the presence of increased inspiratory load, these spindles could provide, via the gamma loop, additional drive to inspiratory ribcage muscles [26], which would help to explain their apparent greater contribution to inspiratory pressure in the CF group. The pressure developed by the inspiratory muscles is also determined by their mass and by their mechanical advantage in converting the tension developed into effective inspiratory pressure. The mass of the diaphragm was reported to be well preserved in CF patients in spite of malnutrition, a finding which was interpreted as reflecting a training response to the increased respiratory load [2]. As the present results suggest, a greater fraction of the inspiratory load was taken up by the inspiratory ribcage muscles in the $\mathrm{CF}$ group. Although initially, this may have been caused by a relatively greater neural activation in response to loading, adaptations at the muscular level could also have been greater in these muscles, perhaps partially explaining their greater contribution to inspiratory pressure. While an elongation of the bony ribcage in CF patients may protect the diaphragm from excessive shortening, it may not prevent changes in its geometry. Indeed, an increase in radius of curvature of the diaphragm can be expected to occur when ribcage diameters increase [27]. According to Laplace's law, this should reduce $P$ di for a given tension $[8,28]$. Several diaphragm dome shape factors were in fact found to be smaller in CF patients than in controls, indicating a flatter diaphragm (table 2). Thus, while an essentially normal Ldi, as seen in female CF patients at FRC, may preserve tension generation in spite of hyperinflation, the gain made may be partially offset by geometric changes caused by ribcage expansion, reducing the pressure developed for a given tension. The fact that ribcage expansion in CF patients occurred without rib elevation (table 2) may also help to explain this finding, as this would tend to preserve the length of the inspiratory ribcage muscles, thereby preserving their inspiratory pressure-generating capacity relative to the diaphragm [29]. An elongation of the ribs, by increasing the change in lung volume associated with a given shortening of the inspiratory ribcage muscles, may also increase their mechanical advantage [29], helping to explain the apparent greater contribution of these muscles to inspiratory pressure, particularly in female CF patients.

In line with earlier findings in patients with chronic obstructive pulmonary disease [30], abdominal muscle recruitment during resting breathing was found in $38 \%$ of the CF patients in the present study. Although the underlying mechanism was not investigated, an increase in chemical drive caused by the added dead space and by the impaired gas exchange in the lungs is likely to contribute. Tidal expiratory flow limitation and increased vagal afferent feedback caused by increased FRC are other factors that may have contributed. The incidence of abdominal muscle recruitment was the same in male and in female CF patients and was thus unlikely to have contributed to the different pattern of thoraco-abdominal adaptation and inspiratory pressure generation observed between males and females. Nevertheless, the contraction of the abdominal muscles during expiration and their relaxation during the subsequent inspiration may have contributed to the reduction of tidal Pdi and Pga swings and hence to the apparent smaller contribution of the diaphragm to inspiratory pressure in the $\mathrm{CF}$ group [31]. However, the comparisons in figure 3 and table 3 were essentially the same, whether patients showing evidence of abdominal muscle recruitment were included or not. Hence, the contribution of abdominal muscle relaxation to inspiratory pressure generation should have been relatively small.

Respiratory failure is the primary cause of death in CF patients. Furthermore, prognosis is substantially poorer in females, with a median survival $3-5$ yrs shorter than in males $[32,33]$. The reason for this sex difference in survival is unclear. However, some of the present findings may be relevant to this discussion. Indeed, the cause of respiratory failure in CF patients is multifactorial and includes worsening ventilation-perfusion inequalities, increased dead space ventilation, increased work and energy cost of breathing and reduced respiratory muscle mechanical efficiency and strength [34]. As mentioned before, changes in diaphragm geometry with ribcage expansion may impair the pressure-generating capacity of the diaphragm, particularly in females in whom ribcage expansion was predominant (fig. 1). In the presence of worsening lung mechanics, increased work of breathing and malnutrition, this factor may eventually contribute to precipitate respiratory failure more quickly in females. The associated reduction in mechanical efficiency of the diaphragm, by increasing the energy cost of breathing and carbon dioxide production in a disproportionate manner, may also promote hypercarpnia more rapidly in females [35]. A relatively greater contribution of the inspiratory ribcage muscles to inspiratory pressure, relative to the diaphragm, as documented in female $\mathrm{CF}$ patients (fig. 3), may also alter ventilation distribution and in this way promote ventilation-perfusion inequalities in the lungs with a consequent worsening hypoxaemia [36]. The same factors may also be expected to impair exercise performance to a greater extent in female than in male CF patients. Clearly, further studies will be required to test these 
possibilities and unravel this important, yet unexplained, sex difference in prognosis in CF patients.

While a structural expansion of the rib cage may have deleterious consequences for the diaphragm and for gas exchange, particularly in female CF patients, it may also carry some benefits. Indeed, both the greater volume capacity of the rib cage relative to the lungs [15] and the longer ribs in relation to height [25] in females than in males have been interpreted as reflecting an evolutionary adaptation for pregnancy that minimises the negative effects of abdominal distension on lung function and abdominal pressure. The structural expansion of the rib cage in female CF patients may thus preserve this advantage in spite of pulmonary hyperinflation, should they become pregnant. This may contribute to explain why pregnancy does not appear to carry additional risks for survival in female CF patients [37].

\section{Conclusion}

In summary, the present study has shown a systematic sex difference in the pattern of thoracic adaptation to pulmonary hyperinflation in cystic fibrosis patients. In female patients, ribcage expansion was predominant, with a consequent relative preservation of diaphragm length. In male patients, a more normal distribution was found between the ribcage and the diaphragm-abdomen, with a consequent greater shortening of the diaphragm. A structural adaptation of the ribcage, presumably owing to an outgrowth of the ribcage 2.5 times greater in females than males, could explain these findings. This structural adaptation of the ribcage appeared to be associated, during quiet breathing, with an enhanced contribution of inspiratory ribcage muscles to inspiratory pressure relative to the diaphragm. These findings may be relevant to the wellknown sex difference in survival in cystic fibrosis patients.

\section{ACKNOWLEDGEMENTS}

The authors wish to thank the personnel of the radiology department at Hôtel-Dieu for their help with chest radiography, L. Lands of the Montreal Children's Hospital for his help with patient recruitment and the Canadian Institutes for Health Research for their support.

\section{REFERENCES}

1 Szeinberg A, England S, Mindorff C, Fraser IA, Levison H. Maximal inspiratory and expiratory pressures are reduced in hyperinflated, malnpourished, young adult male patients with cystic fibrosis. Am Rev Respir Dis 1985; 132: 766-769.

2 Pinet C, Cassart M, Scillia P, et al. Function and bulk of respiratory and limb muscles in patients with cystic fibrosis. Am J Respir Crit Care Med 2003; 168: 989-994.

3 Coates AL, Boyce P, Muller D, Mearns M, Godfrey S. The role of nutritional status, airway obstruction, hypoxia, and abnormalities in serum lipid composition in limiting exercise tolerance in children with cystic fibrosis. Am Rev Respir Dis 1982; 126: 855-859.

4 Braun NM, Arora NS, Rochester DF. Force-length relationship of the normal human diaphragm. J Appl Physiol 1982; 53: 405-412.

5 Rochester DF, Braun NMT. Determinants of maximal inspiratory pressure in chronic obstructive pulmonary disease. Am Rev Respir Dis 1985; 132: 42-47.
6 Cassart M, Pettiaux N, Gevenois PA, Paiva M, Estenne M. Effect of chronic hyperinflation on diaphragm length and surface area. Am J Respir Crit Care Med 1997; 156: 504-508.

7 Bellemare F, Cordeau MP, Couture J, Lafontaine E, Leblanc P, Passerini L. Effects of emphysema and lung volume reduction surgery on transdiaphragmatic pressure and diaphragm length. Chest 2002; 121: 1898-1910.

8 Marshall R. Relationship between stimulus and work of breathing at different lung volumes. J Appl Physiol 1962; 17: 917-921.

9 Lands L, Desmond KJ, Demizio D, Pavilanis A, Coates AL. The effects of nutritional status and hyperinflation on respiratory muscle strength in chidren and young adults. Am Rev Respir Dis 1990; 141: 1506-1509.

10 Pradal U, Polese G, Braggion C, et al. Determinants of maximal transdiaphragmatic pressure in adults with cystic fibrosis. Am J Respir Crit Care Med 1994; 150: 167-173.

11 Lands LC, Heigenhauser GJF, Jones NL. Respiratory and peripheral muscle function in cystic fibrosis. Am Rev Respir Dis 1993; 147: 865-869.

12 O'Neill S, Leahy F, Pasterkamp H, Tal A. The effects of chronic hyperinflation, nutritional status, and posture on respiratory muscle strength in cystic fibrosis. Am Rev Respir Dis 1983; 128: 1051-1054.

13 Bellemare JF, Cordeau MP, Leblanc P, Bellemare F. Thoracic dimensions at maximum lung inflation in normal subjects and in patients with obstructive and restrictive lung diseases. Chest 2001; 119: 376-386.

14 Bellemare F, Couture J, Cordeau MP, Leblanc P, Lafontaine E. Anatomic landmarks to estimate the length of the diaphragm from chest radiographs: effects of emphysema and lung volume reduction surgery. Chest 2001; 120: 444-452.

15 Bellemare F, Jeanneret A, Couture J. Sex differences in thoracic dimensions and configuration. Am J Respir Crit Care Med 2003; 168: 305-312.

16 Pierce RJ, Brown DJ, Holmes M, Cumming G, Denison DM. Estimation of lung volumes from chest radiographs using shape information. Thorax 1979; 34: 726-734.

17 Singh B, Panizza JA, Finucane KE. Breath-by-breath measurement of the volume displaced by diaphragm motion. J Appl Physiol 2003; 94: 1084-1091.

18 Loring S, Mead J, Griscom NT. Dependence of diaphragmatic length on lung volume and thoracoabdominal configuration. J Appl Physiol 1985; 59: 1961-1970.

19 Coates AL, Davis GM, Vallinis P, Outerbridge EW. Liquidfilled esophageal catheter for measuring pleural pressure in preterm neonates. J Appl Physiol 1989; 67: 889-893.

20 Macklem PT, Gross D, Grassino A, Roussos C. Partitioning of inspiratory pressure swings between diaphragm and intercostal/accessory muscles. J Appl Physiol 1978; 44: 200-208.

21 Wilson TA, Rehder K, Krayer S, Hoffman EA, Whitney CG, Rodarte JR. Geometry and respiratory displacement of human ribs. J Appl Physiol 1987; 62: 1872-1877.

22 Thomas AJ, Supinski GS, Kelsen SG. Changes in chest wall structure and elasticity in elastase-induced emphysema. J Appl Physiol 1986; 61: 1821-1829.

23 DeMuth GR, Howatt WF, Hill BM. Growth of lung function. Part 1. Lung volumes. Part 2. Configuration of the chest. Pediatrics 1965; 35: 162-184. 
24 Guignon I, Cassart M, Gevenois PA, et al. Persistent hyperinflation after heart-lung transplantation for cystic fibrosis. Am J Respir Crit Care Med 1995; 151: 534-540.

25 Bellemare F, Fuamba T, Bourgeault A. Sexual dimorphism of human ribs. Respir Physiol Neurobiol 2006; 150: 233-239.

26 Sears T. Efferent discharges in alpha and fusimotor fibres of intercostal nerves of the cat. J Physiol 1964; 174: 295-315.

27 Gauthier AP, Verbanck S, Estenne M, Segebarth C, Macklem PT, Paiva M. Three-dimensional reconstruction of the in vivo human diaphragm shape at different lung volumes. J Appl Physiol 1994; 76: 495-506.

28 Paiva M, Verbanck S, Estenne M, Poncelet B, Segebarth C, Macklem PT. Mechanical implications of in vivo human diaphragm shape. J Appl Physiol 1992; 72: 1407-1412.

29 Wilson TA, Legrand A, Gevenois PA, Troyer AD. Respiratory effects of the external and internal intercostal muscles in humans. J Physiol 2001; 530: 319-330.

30 Ninane V, Rypens F, Yernault JC, De Troyer A. Abdominal muscle use during breathing in patients with chronic airflow obstruction. Am Rev Respir Dis 1992; 146: 16-21.
31 De Troyer A. Mechanical role of the abdominal muscles in relation to posture. Respir Physiol 1983; 53: 341-353.

32 Corey M, Farewell V. Determinants of mortality from cystic fibrosis in Canada,1970-1989. Am J Epidemiol 1996; 143: 1007-1017.

33 Rosenfeld M, Davis R, FitzSimmons S, Pepe M, Ramsey B. Gender gap in cystic fibrosis mortality. Am J Epidemiol 1997; 145: 794-803.

34 Davis PB. Clinical pathophysiology and manifestations of lung disease. In: Yankaskas JR, Knowles MR, eds. Cystic Fibrosis in Adults. Philadelphia, Lippincott-Raven, 1999; pp. $45-67$.

35 Weiner P, Suo J, Fernandez E, Cherniack RM. Efficiency of the respiratory muscles in healthy individuals. Am Rev Respir Dis 1989; 140: 392-396.

36 Roussos CS, Fixley M, Genest J, et al. Voluntary factors influencing the distribution of inspired gas. Am Rev Respir Dis 1977; 116: 457-467.

37 Goss CH, Rubenfeld GD, Otto K, Aitken ML. The effect of pregnancy on survival in women with cystic fibrosis. Chest 2003; 124: 1460-1468. 